\title{
Estrategias para el aprendizaje significativo en instituciones de Educación Preescolar
}

\author{
Strategies for meaningful learning in preschool institutions
}

\author{
Yasmin Pineda Ariza \\ yasmindarem@hotmail.com \\ Código ORCID: 0000-0002-6932-7533 \\ Institución educativa Rodrigo \\ Galván de la Bastidas, Colombia
}

\author{
Maigualida Bejas Monzant \\ maigualidabejas@yahoo.com \\ Código ORCID: 0000-0003-1859-1948 \\ Universidad del Zulia, Venezuela
}

\section{RESUMEN}

- Artículo recibido en octubre 2019 - Arbitrado en noviembre 2019 - Publicado en enero 2020

La investigación tuvo como objetivo analizar las estrategias que utilizan las docentes para el aprendizaje significativo en instituciones de educación preescolar. Metodológicamente se enmarca dentro del enfoque cuantitativo, el tipo de investigación es descriptiva con un diseño no experimental y de campo, la población estuvo conformada por once docentes del nivel preescolar, la muestra es de tipo censal, a los cuales se les aplicó un cuestionario. La validez de los mismos se llevó a cabo a través del juicio de cinco expertos y la confiabilidad se determinó aplicando el Coeficiente del Alpha-Cronbach, el cual arrojo un valor de 0,947. Se concluyó que esta investigación puede generar en las docentes y en la misma institución educativa un interés por profundizar en el análisis de las estructuras pedagógicas organizadas en los planes de estudios, especialmente del nivel preescolar.

Palabras clave: Estrategias; estrategias de enseñanza; aprendizaje significativo: educación preescolar

\section{ABSTRACT}

The research aimed to analyze the strategies that teachers use for meaningful learning in preschool institutions. Methodologically is framed within the quantitative approach, the type of research is descriptive with a non-experimental and field design, the population was made up of eleven teachers at the preschool level, the sample is of the census type, to which a questionnaire. The validity of the same was carried out through the judgment of five experts and the reliability was determined by applying the Alpha-Cronbach coefficient, which yielded a value of 0.947. It was concluded that this research can generate an interest in the teachers and in the educational institution itself to deepen the analysis of the pedagogical structures organized in the curricula, especially at the preschool level.

Keywords: Strategies; teaching strategies; meaningful learning: preschool education 


\section{INTRODUCCIÓN}

La situación actual en el sector educativo de Colombia es preocupante $\mathrm{y}$ aunque en algunos entes gubernamentales y no gubernamentales del país se vienen haciendo esfuerzos importantes por mejorar la calidad educativa, estos no han sido suficientes. La baja calidad de la educación es algo que no puede negarse, se evidencia desde la educación preescolar y en los resultados de las evaluaciones que se aplican periódicamente para monitorear el desarrollo de las competencias en los estudiantes de educación básica, como seguimiento de calidad del sistema educativo colombiano (pruebas saber) y las pruebas Pisa de manera categórica.

Según, Alvarado y Suárez (2014) en el documento de política "Colombia por la primera infancia", se puede resaltar que a pesar que los procesos en la educación preescolar están orientados en diferentes propuestas y utilizando diversas estrategias, para lograr esa articulación con la educación básica aún no hay una claridad sobre cuáles podrían ser los mecanismos que garanticen dicha articulación. Sin duda, los beneficios que brinda la educación preescolar son evidentes, y no puede desconocerse su importancia como primer pasó cuando se piensa en una formación educativa de calidad niña y niña.

La problemática que se plantea podría tener su origen en aspectos como: la falta de idoneidad docente, para su trabajo en el aula, en relación a enfoques tradicionales con estrategias poco significativas para los estudiantes. Las instituciones educativas en el Distrito de Santa Marta no son ajenas a esta situación, es así como este planteamiento se convierte en el objeto central de esta investigación el cual es la aplicación de estrategias de enseñanzas para el aprendizaje significativo por parte de las docentes de educación preescolar en las instituciones educativas, para lo cual tomaremos como referente la institución educativa distrital Rodrigo Galván de la Bastidas.

La carencia de recursos didácticos motivadores e innovadores, de ambientes adecuados para el desarrollo del potencial de aprendizaje, la falta de una motivación apropiada que estimule la creatividad, la imaginación, el desarrollo de habilidades cognitivas y motrices, son también aspectos relevantes que influyen en la manera como las docentes desarrollan sus procesos de enseñanza, según Mieles y col (2009), en las docentes de preescolar del Distrito existe compromiso y responsabilidad frente a la tarea educativa. Sin embargo algunos aspectos deben ser reflexionados $\mathrm{y}$ mejorados como la pasividad, la rutina y la desactualización que junto con las condiciones reales de las instituciones educativas, impiden que el proceso formativo de los niños y niñas sea el adecuado. 
Adicional a lo anterior, la poca relevancia que se le da a este nivel y la insuficiente apropiación por parte de los directivos y docentes en relación con las políticas en las cuales está enmarcado el nivel preescolar, constituye otro factor de gran importancia en la problemática planteada.

Según, investigaciones realizadas por el Grupo de Investigación en Educación Infantil de la Universidad del Magdalena en el año 2009, el cual concluyó que el $100 \%$ de los directivos docentes consideran que es poco valorada y el $2 \%$ de docentes de otras áreas que es muy valorada, además muchos docentes del nivel preescolar no son licenciados en educación preescolar, otras no han continuado con procesos de educación e investigación permanente o son muy incipientes $o$ no se realizan, lo cual evidencia la subvaloración que se tiene de los procesos de formación en el grado transición.

Si se hace un análisis de los elementos expuestos en la problemática de la presente investigación, esto genera una serie de factores que influyen directamente en el proceso de aprendizaje significativo, relacionados con la inadecuada preparación que tendrían los niños y niñas, lo cual no les permitiría enfrentarse apropiadamente a las exigencias iniciales del nivel básico, al respecto León (2010) expresa su opinión, basado en una apreciación de la Unesco (2007), la cual plantea que los niños y niñas al ingresar a su primer grado escolar de básica primaria deben manejar una serie de condiciones que se convierten en requisitos para el éxito.

Todas las situaciones descritas anteriormente en relación al poco uso de estrategias pedagógicas por parte de las docentes de preescolar, unido a otros factores nos lleva a pensar que probablemente en algunos años la educación preescolar perderá su importancia como eje fundamental y central en la preparación de los niños y niñas de una manera apropiada para la iniciación de la educación básica.

Por tanto, el objetivo general del presente estudio fue: Analizar las estrategias de aprendizaje significativo que utilizan las docentes en las instituciones de educación preescolar. En cuanto a los objetivos específicos: Identificar las estrategias pre instruccionales que utilizan las docentes de para el aprendizaje significativo en instituciones de educación preescolar; describir las estrategias coinstruccionales que utilizan las docentes para el aprendizaje significativo en instituciones de educación preescolar, $\mathrm{y}$, por último, caracterizar las estrategias posinstruccionales que utilizan las docentes de para el aprendizaje significativo en instituciones de educación preescolar.

La concepción conceptual de la variable estrategia de aprendizaje significativo presenta diversas clasificaciones, pero centro en la 
realizada por Díaz Barriga y Hernández (2002) basadas en su momento de uso y presentación, pues son de gran interés para sustentar la presente investigación Estrategias Preinstrucionales. Según Díaz Barriga y Hernández (2002) son todas aquellas que se utilizan al inicio de una sesión; por lo general predisponen positivamente al estudiante en relación al qué y cómo va a aprender además que tratan de incidir en la activación de conocimientos o de generarlos si no existieran, situando además al aprendiz en el contexto conceptual adecuado y crea expectativas apropiadas Enfoque de la Investigación.

Así mismo, Díaz-Barriga y Hernández (2002) afirman que estas estrategias tienen la intención de ayudar al estudiante a mejorar la atención, seleccionar la información central para una mejor codificación y mayor contextualización de los contenidos de aprendizaje, organizar y estructurar las ideas relevantes.

Las estrategias construccionales muestran su influencia también en los procesos curriculares, pues en su desarrollo y aplicación correcta cumplen funciones que pueden ser relevantes a la hora de manejar los diversos conceptos o contenidos vistos. De acuerdo a lo anterior Campos (2000) expresa que estas estrategias proporcionan la oportunidad para que el estudiante realice una codificación complementaria a la realizada por el profesor, o por el texto, con la intención de enriquecer la información nueva al contar con una mayor contextualización para una mejor abstracción de modelos conceptuales.

$\mathrm{Y}$ las estrategias posinstruccionales son aquellas que se presentan después del contenido que se ha de aprender y permiten al estudiante formar una visión sintética integradora del material propuesto. En tal sentido, Díaz-Barriga y Hernández (2002) plantean que estas permiten incluso una visión crítica del material, lo que favorece que el estudiante analice y valore su propio aprendizaje al ver el producto de su esfuerzo y dedicación. Con estas estrategias no solo se logra constatar por sí mismo su avance, sino qué pretendió desde el inicio, además que el docente pueda estar satisfecho que realmente la clase fue productiva.

Estas estrategias sirven también para organizar la información nueva. Para Villarruel (2009) son reservadas a promover y potenciar enlaces adecuados entre los conocimientos previos y la información nueva (andamiaje), asegurando con ellas un mayor significado en los aprendizajes lo que permite que la información nueva que se va a aprender se organice correctamente, con base en determinados contextos o escenarios de aprendizaje. Pueden presentarse en forma gráfica o escrita; la idea es dotar de significancia al proceso de aprendizaje. 


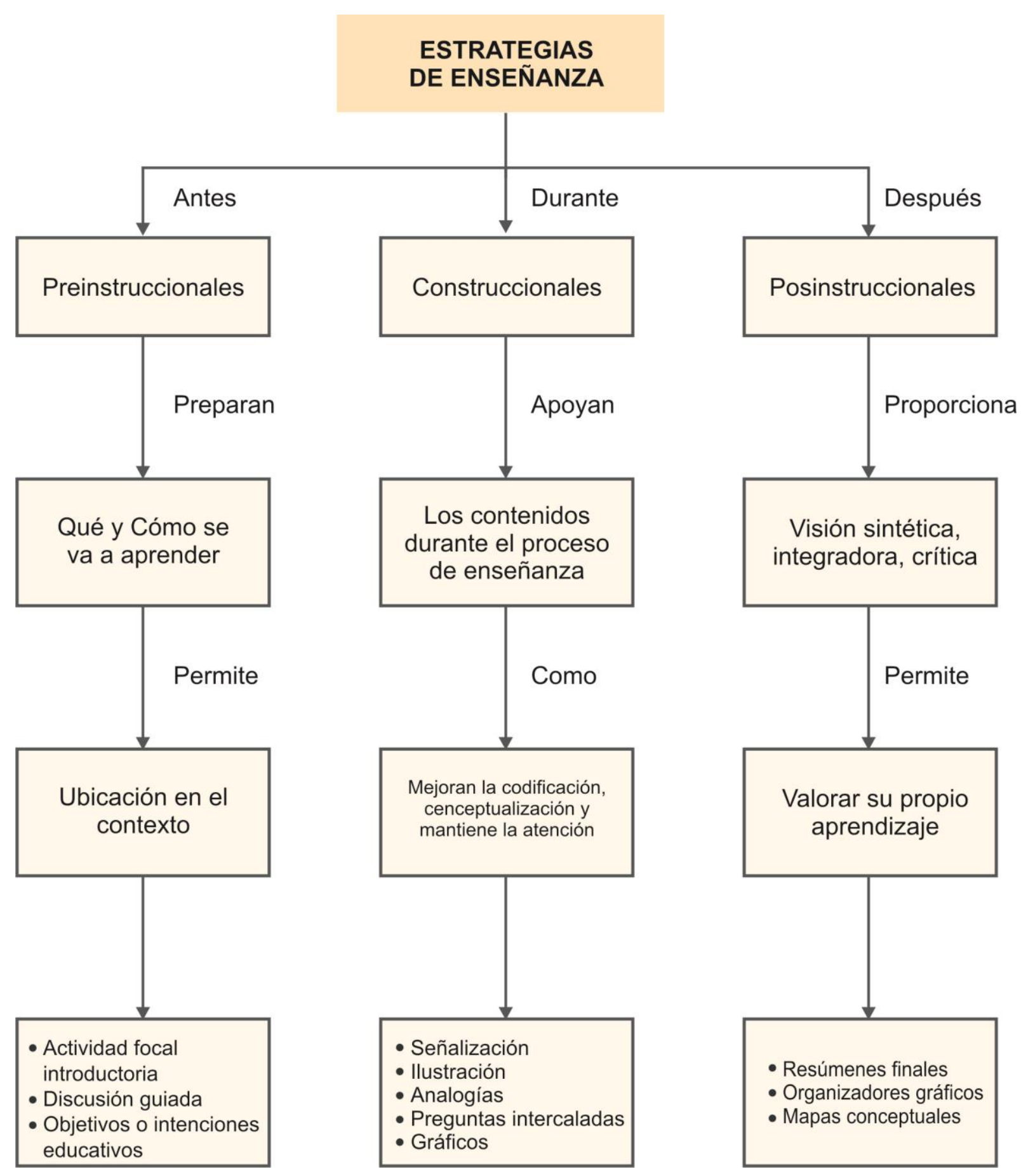

Figura 1. Estrategias de enseñanza (Fuente: Díaz Barriga y Hernández, 2002). 


\section{MÉTODO}

La investigación se enmarcó dentro del enfoque cuantitativo, debido que esta plantea un problema de estudio delimitado y concreto y la pregunta de investigación trató de un tema específico, además se utiliza la recolección de datos fundamentados en la medición numérica analizados a través de métodos estadístico para dar respuesta a los objetivos planteados. El tipo de investigación fue descriptiva, puesto que en este caso solo se limita a describir las características de la variable en estudio tal como suceden en la realidad abordada. El diseño de la investigación fue de campo no experimental por cuanto los datos serán recogidos en el mismo lugar, es decir que serán suministrados directamente por los participantes en su propio contexto.

La población de la investigación se conformó por 34 docentes del nivel preescolar de la localidad 2 Histórico Rodrigo de Bastidas de Santa Marta, que cuenta con cinco sedes (ver Tabla 1). En la investigación se utilizó además una una población documental, pues abordará el análisis de una muestra de los planes de aula realizados por los docentes de preescolar de la Institución Educativa Distrital Rodrigo Galván de la Bastidas.

Tabla 1. Población: Docentes de preescolar de la localidad

\begin{tabular}{|c|c|}
\hline SEDES & $\mathrm{N}^{\circ}$ DE DOCENTES DE PREESCOLAR \\
\hline I.ED. Rodrigo Galván de la Bastidas & 11 \\
\hline I.ED. Ondas Del Caribe & 7 \\
\hline I.ED. Pantano & 3 \\
\hline I.ED. Nicolás Buenaventura & 6 \\
\hline I.ED. Rodrigo de Bastidas & 7 \\
\hline Total & 34 \\
\hline
\end{tabular}

Fuente: Elaboración propia.

La muestra de la investigación, según Martínez (2012) indica que "En el muestreo no probabilístico se toma la muestra de cualquier tamaño y los elementos son seleccionados de acuerdo con la opinión o juicio que tenga el investigador sobre la población. En el caso de una población homogénea, la representatividad de tal muestra puede considerarse satisfactoria" ( $p$ 18). Para este caso, los principales criterios que privaron en la selección de la muestra fueron el tiempo y los recursos disponibles y las características de los docentes que están referidas a; pertenecer al área de estudio de preescolar, y desarrollan el nivel de iniciación de la escuela para realizar la investigación. Tomando en cuenta estos criterios, se usó el muestreo intencional $\mathrm{u}$ opinático, siendo la muestra seleccionada la siguiente 
Tabla 2. Distribución de la Muestra de la investigación. Institución educativa Distrital Rodrigo Galván de la Bastidas

\begin{tabular}{ll}
\hline SEDES & $\mathbf{N}^{\circ}$ DE DOCENTES \\
\hline $\mathrm{N}^{\circ}$ 1 Principal & 4 \\
$\mathrm{~N}^{\circ}$ 2 Tayrona & 3 \\
$\mathrm{~N}^{\circ}$ 3 Villa Del Rio & 2 \\
$\mathrm{~N}^{\circ}$ 4 Simón Bolívar & 2 \\
Total & $\mathbf{1 1}$ \\
\hline
\end{tabular}

Fuente: Elaboración propia.

La muestra de la investigación, según Martínez (2012) indica que "En el muestreo no probabilístico se toma la muestra de cualquier tamaño y los elementos son seleccionados de acuerdo con la opinión o juicio que tenga el investigador sobre la población. En el caso de una población homogénea, la representatividad de tal muestra puede considerarse satisfactoria" ( $p$ 18). Para este caso, los principales criterios que privaron en la selección de la muestra fueron el tiempo y los recursos disponibles y las características de los docentes que están referidas a; pertenecer al área de estudio de preescolar, y desarrollan el nivel de iniciación de la escuela para realizar la investigación. Tomando en cuenta estos criterios, se usó el muestreo intencional u opinático, siendo la muestra seleccionada la siguiente:

Tabla 3. Estructura del Instrumento (Cuestionario)

\begin{tabular}{|c|c|c|c|c|c|}
\hline VARIABLE & DIMENSIÓN & INDICADORES & ITEMS & $\begin{array}{c}\mathbf{N}^{\circ} \\
\text { ITEMS }\end{array}$ & TOTAL \\
\hline \multirow{14}{*}{$\begin{array}{l}\text { Estrategias } \\
\text { para el } \\
\text { aprendizaje } \\
\text { significativo }\end{array}$} & \multirow{6}{*}{ Preinstrucionales } & Lluvia de ideas & $1,2,3$ & 3 & \multirow[t]{6}{*}{15} \\
\hline & & $\begin{array}{l}\text { Actividad focal } \\
\text { introductoria }\end{array}$ & $4,5,6$ & 3 & \\
\hline & & Discusión guiada & $7,8,9$ & 3 & \\
\hline & & $\begin{array}{l}\text { objetivos } \\
\text { intenciones } \\
\text { educativos }\end{array}$ & $10,11,12$ & 3 & \\
\hline & & $\begin{array}{l}\text { Organizadores } \\
\text { previos }\end{array}$ & $13,14,15$ & 3 & \\
\hline & & Señalizaciones & $16,17,18$ & 3 & \\
\hline & \multirow[b]{3}{*}{ Coinstrucionales } & Ilustraciones & $19.20,21$ & 3 & \\
\hline & & Analogías & $22,23,24$ & 3 & \\
\hline & & $\begin{array}{l}\text { preguntas } \\
\text { intercaladas }\end{array}$ & $25,26,27$ & 3 & \\
\hline & \multirow{4}{*}{ Posinstrucionales } & Graficas & $28,29,30$ & 3 & \multirow{4}{*}{9} \\
\hline & & Resúmenes & $31,32,33$ & 3 & \\
\hline & & $\begin{array}{l}\text { Organizadores } \\
\text { gráficos }\end{array}$ & $34,35,36$ & 3 & \\
\hline & & Mapas conceptuales & $37,38,39$ & 3 & \\
\hline & Total & & & & 39 \\
\hline
\end{tabular}

Fuente: Elaboración propia. 
La validez y confiabilidad del instrumento se determinó en este estudio se utilizó el coeficiente Alfa de Cronbach, debido a que el instrumento tiene varias alternativas de respuesta, cuya expresión matemática es la siguiente:

$$
\begin{aligned}
& \alpha=\left(\frac{p}{p-1}\right)\left(1-\frac{\sum_{j=1}^{p} S_{j}^{2}}{S_{t}^{2}}\right) \\
&
\end{aligned} \quad \begin{aligned}
& \text { Dónde: } \\
& \mathrm{S}_{\mathrm{j}}^{2} \text { : Es la varianza de cada ítem. } \\
& \mathrm{S}_{\mathrm{t}}^{2} \text { : Es la varianza de la suma de los ítems. } \\
& \mathrm{p}: \text { Es el total de ítems } \\
& \mathrm{r}_{\mathrm{p}} \text { : Es el promedio de las correlaciones entre de los ítems }
\end{aligned}
$$

Al aplicar el paquete estadístico SPSS, versión 19, a la base de datos de los 39 ítems, se obtuvo para el coeficiente Alfa de Cronbach un valor de $\alpha=0,947$. Este resultado indica que el instrumento diseñado es altamente confiable.

\section{RESULTADOS}

Es válido destacar en sección del artículo que en la presente investigación no aplicó el análisis estadístico de la masa de datos, derivado de la introducción de medidas de tendencia central, índices de variación o medidas de dispersión, para establecer los resultados. Los datos recolectados, atendiendo a las variables de estudio, fueron tratados y resumidos a través del uso de porcentajes, manejados a partir de las convenciones admitidas universalmente. Para este propósito, se utilizó el Programa SPSS, versión 19 estándar para Windows, el cual es un software estadístico cuyas siglas, traducidas al español, significan Paquete
Estadístico para las Ciencias Sociales y que está construido específicamente para el análisis estadístico y la gestión de datos.

Al introducir los porcentajes en el análisis de los datos, se trata de proporciones que se multiplican por 100 . En la medida que estas proporciones expresan los valores de cada variable analizada en función del valor general del universo en estudio.

En este orden de ideas, el proceso técnico estadístico que se efectuó en esta investigación, estuvo centrado Análisis del Cuestionario Autoadministrado. Se basó, fundamentalmente, en la Estadística Descriptiva, obteniéndose a través del programa estadístico SPSS versión 19, los siguientes insumos que fueron de gran utilidad en el estudio del instrumento antes citado: Tablas de Distribución de Frecuencias y Diagramas de Barras; todo esto de cada uno de los ítems que conforman los instrumentos. 
Por otra parte, para el estudio de cada uno de los indicadores se tomó el promedio de los ítems que conformaron cada indicador según lo estipulado en la Tabla de operacionalización teórica de las variables, también para el estudio de las dimensiones se tomó el promedio de los indicadores que la conformaron y para el estudio de la variable se tomó en cuenta el promedio de las dimensiones que la conforman. Dado que los resultados estuvieron entre el valor mínimo que en este caso es el correspondiente a Nunca (1) y el valor máximo que es el correspondiente a Siempre (5), al tener en cuenta que en algunos casos los resultados no serán números naturales, surge la necesidad de diseñar un baremo que tipifique todos los posibles resultados en la escala original, para su posterior análisis. Dicho baremo se presenta a continuación:

Tabla 4. Baremo de Transformación Escala de los Indicadores, Dimensiones y Variable.

\begin{tabular}{ccc}
\hline RANGO & ALTERNATIVA & ESCALA \\
\hline $5,00-4,20$ & SIEMPRE & 5 \\
$4,19-3,39$ & CASI SIEMPRE & 4 \\
$3,38-2,58$ & ALGUNAS VECES & 3 \\
$2,57-1,77$ & CASI NUNCA & 2 \\
$1,76-1,00$ & NUNCA & 1 \\
\hline
\end{tabular}

Fuente: Elaboración propia.

Para el análisis de las frecuencias, se consideraron como favorables las opciones Siempre (5) y Casi Siempre (4), tomándose en cuenta la suma de sus frecuencias porcentuales. Se tomó como tendencia favorable cuando este porcentaje fue mayor o igual al $80 \%$. Debe entenderse como tendencia favorable, la inexistencia estadísticamente significativa de carencias notables en el aspecto que se considera por parte de las unidades de análisis (Sujetos de investigación).

Este análisis se realizó para cada indicador, dimensión y variable: Para cada indicador, se determinó el promedio de los ítems que lo determinan; el análisis de las dimensiones se hizo obteniendo el promedio de los indicadores que las conforman; y para el análisis de la variable de estudio de esta investigación, se Resultados por Dimensiones; Dimensión Pre instruccionales: Los porcentajes de respuestas favorables que muestran la tabla y gráfico que anteceden arrojan un $45,5 \%$ de respuestas consideradas como favorables, valor que al ser comparado con el porcentaje mínimo establecido (80\%) conduce a afirmar que, en general, el grupo de docentes encuestados no muestra una tendencia favorable en la utilización de las estrategias didácticas pre instruccionales. 
Es importante destacar que este resultado es desalentador, puesto que las estrategias didácticas referidas a esta dimensión son de vital importancia para el desarrollo de las actividades que deben realizar los estudiantes para el logro de sus aprendizajes, puesto que se tratan de las "actividades preparatorias" o iniciales del proceso educativo.

Tabla 5. Dimensión preinstruccionales

\begin{tabular}{cccc}
\hline Categoría de respuesta & Frecuencia & Porcentaje & $\begin{array}{c}\text { Porcentaje } \\
\text { acumulado }\end{array}$ \\
\hline 2.00 & 3 & 27.3 & \\
3.00 & 3 & 27.3 & 27.3 \\
4.00 & 2 & 18.2 & 54.5 \\
5.00 & 3 & 27.3 & 72.7 \\
Total & 11 & 100.0 & 100.0 \\
\hline
\end{tabular}

Fuente: SPSS aplicado a la base de datos (2017).

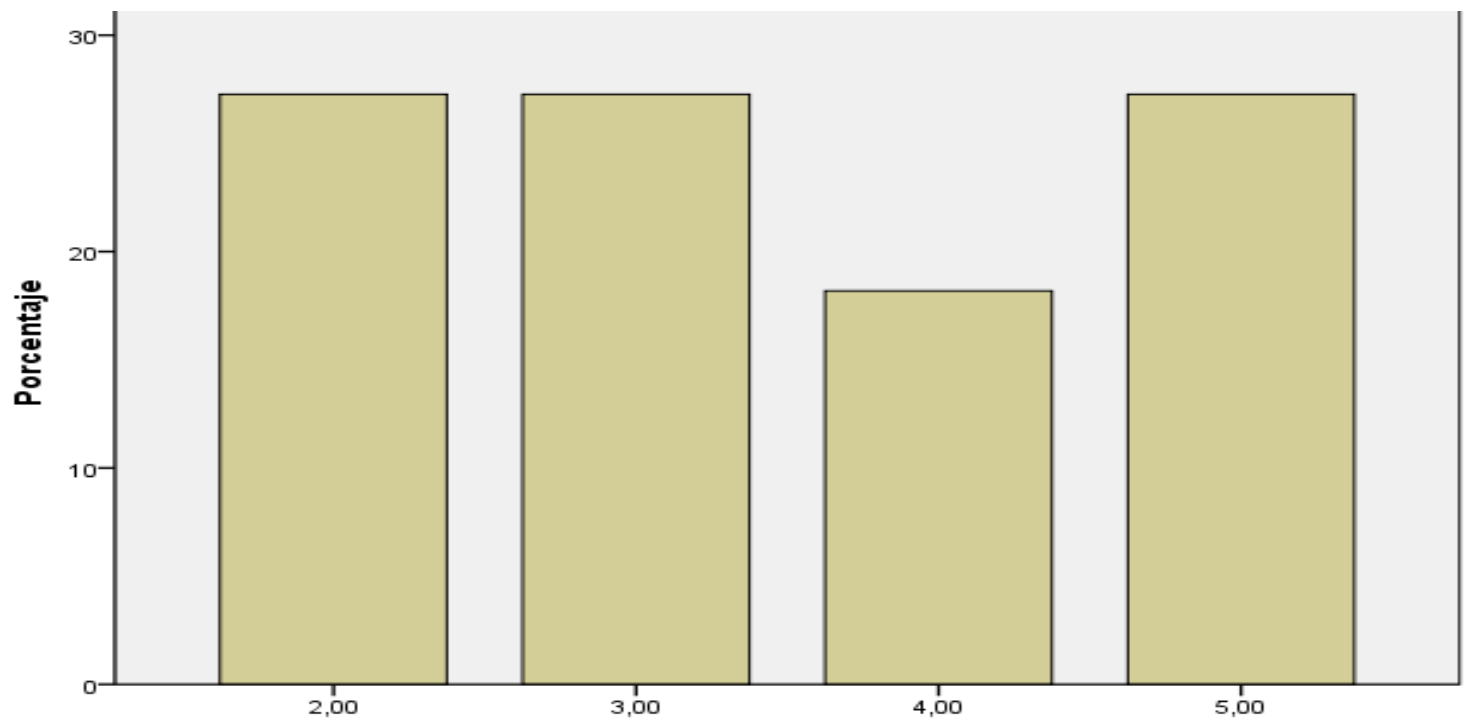

Gráfico 1. Dimensión Preinstruccionales.

Los porcentajes de respuestas muestra una tendencia favorable en la favorables que muestran la Tabla y utilización de las estrategias didácticas gráfico que anteceden arrojan un 45,5\% de respuestas consideradas como favorables, valor que al ser comparado con el porcentaje mínimo establecido $(80 \%)$ conduce a afirmar que, en general, el grupo de docentes encuestados no preinstruccionales. Es importante destacar que este resultado es desalentador, puesto que las estrategias didácticas referidas a esta dimensión son de vital importancia para el desarrollo de las actividades que deben realizar los 
estudiantes para el logro de sus aprendizajes, puesto que se tratan de las "actividades preparatorias" o iniciales del proceso educativo.

Lo anterior contrasta con el planteamiento de Ausubel (1983), el cual expresa que una de las condiciones para que el aprendizaje sea significativo es que la estructura cognoscitiva previa del individuo debe poseer ideas relevantes para luego unirla con el nuevo conocimiento, es decir que antes de abordar un tema debe utilizar estrategias que activen los conocimientos previos de sus estudiantes.

De lo cual se puede concluir que para las docentes de preescolar de la institución objeto de estudio no es relevante la activación de conocimientos previos, según lo demuestran los resultados de la encuesta, denotándose así la carencia de aprendizajes realmente significativos, mostrando más apego a las formas tradicionales de enseñanza, donde el niño o niña es considerado meramente un aprendiz que necesita ser llenado de conocimientos y conceptos básicamente memorísticos.

La activación de dichos conocimientos previos es una condición como se expresa anteriormente para que se dé un aprendizaje significativo. Por otra parte, las docentes no se preocupan por conocer lo que los niños saben acerca del tema que pretenden enseñar, lo que es realmente importante, con lo cual podría incurrirse en el error de repetir conceptos que ya los estudiantes poseen en sus estructuras cognitivas, lo que lleva a que el interés de los niños por el nuevo aprendizaje se pierda o adquiera un valor meramente académico y obligatorio para avanzar en sus procesos escolares.

Dimensión Coinstruccionales: Contrariamente al resultado observado en la dimensión anterior, el porcentaje que se obtuvo para esta dimensión es altamente satisfactorio: 90,9 de respuestas correspondientes a las categorías Siempre y Casi Siempre. Este valor es superior al porcentaje mínimo exigido en el Capítulo precedente, resultado que indica la existencia de una tendencia favorable en el uso de estrategias didácticas referidas a la dimensión Coinstruccionales. Si a este valor agregamos el hecho de la ausencia de respuestas desfavorables (Nunca y Casi Nunca) se evidencia un comportamiento altamente satisfactorio de los docentes del grupo de estudio en la aplicación de este tipo de estrategias didácticas. 
Tabla 6. Dimensión Coinstruccionales

\begin{tabular}{cccc}
\hline Categoría de Respuesta & Frecuencia & Porcentaje & Porcentaje acumulado \\
\hline 3.00 & 1 & 9.1 & 9.1 \\
4.00 & 9 & 81.8 & 90.9 \\
5.00 & 1 & 9.1 & 100.0 \\
Total & 11 & 100.0 & \\
\hline
\end{tabular}

Fuente: SPSS aplicado a la base de datos (2017).

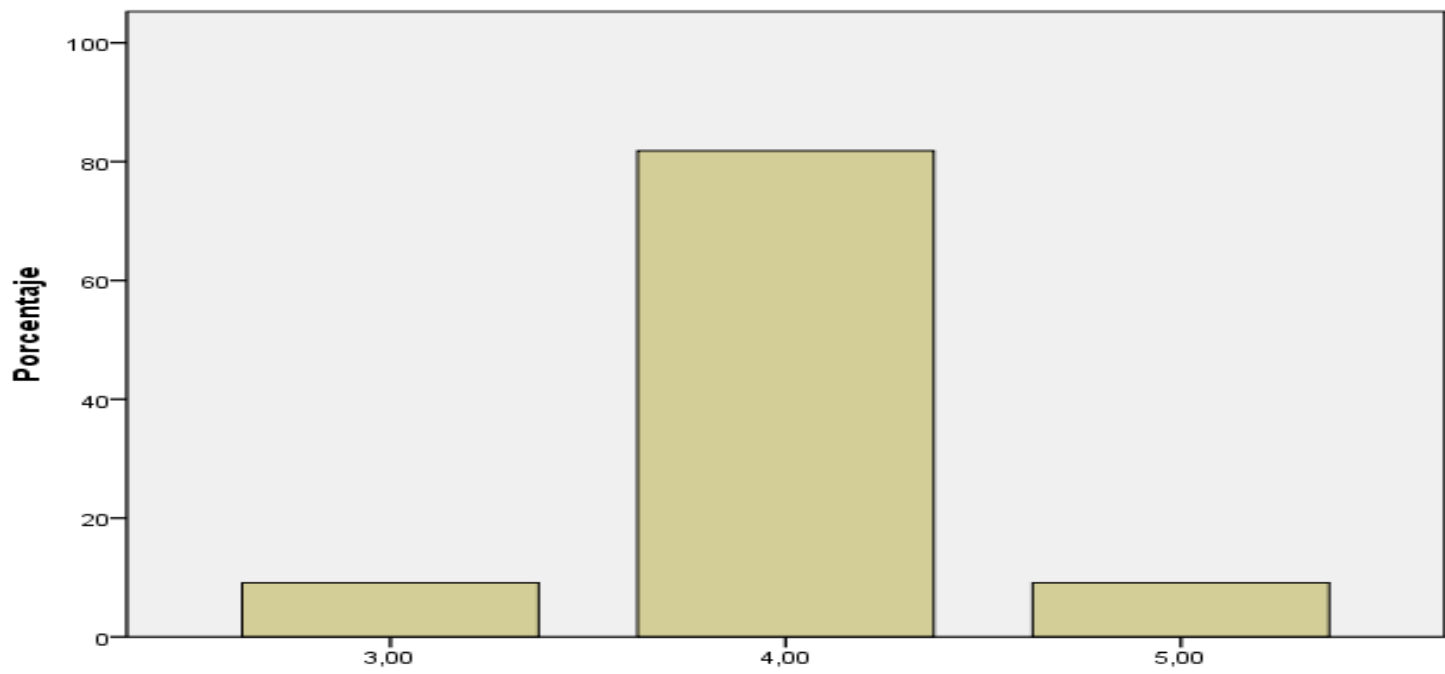

Gráfico 2. Dimensión Coinstruccionales.

Al utilizar en mayor frecuencia el tipo de estrategias coinstruccionales, las docentes reafirman el planteamiento de Díaz Barriga y Hernández (2002) que explican que estas estrategias tienen la intención de ayudar al estudiante a mejorar su atención y a seleccionar la información para una mayor contextualización de los contenidos en cuanto a las ideas más relevantes.

Lo anterior evidencia la preocupación por las docentes de preescolar por utilizar estrategias que ayuden al estudiante a interpretar de manera más adecuada la información recibida con el fin de que estos la apropien a su contexto de ahí que utilicen en gran medida las analogías y las ilustraciones, tal vez por considerar que son de fácil utilización y más apropiadas para la edad de los niños del nivel preescolar, así mismo podría inferirse que estas consideran que es de suma importancia esta manera de presentar el desarrollo de los contenidos $\mathrm{y}$ puede ser suficiente para generar aprendizajes significativos, ya que estas pueden utilizarse durante el desarrollo de toda la clase. 


\section{Dimensión Postinstruccionales:}

Tabla 7. Dimensión Posinstruccionales

\begin{tabular}{llll}
\hline Categoría de Respuesta & Frecuencia & Porcentaje & Porcentaje acumulado \\
\hline & 1 & 9.1 & \\
1.00 & 4 & 36.4 & 4.1 \\
3.00 & 2 & 18.2 & 63.6 \\
4.00 & 3 & 27.3 & 90.9 \\
5.00 & 1 & 9.1 & 100.0 \\
Total & 11 & 100.0 & \\
\hline
\end{tabular}

Fuente: SPSS aplicado a la base de datos (2017).

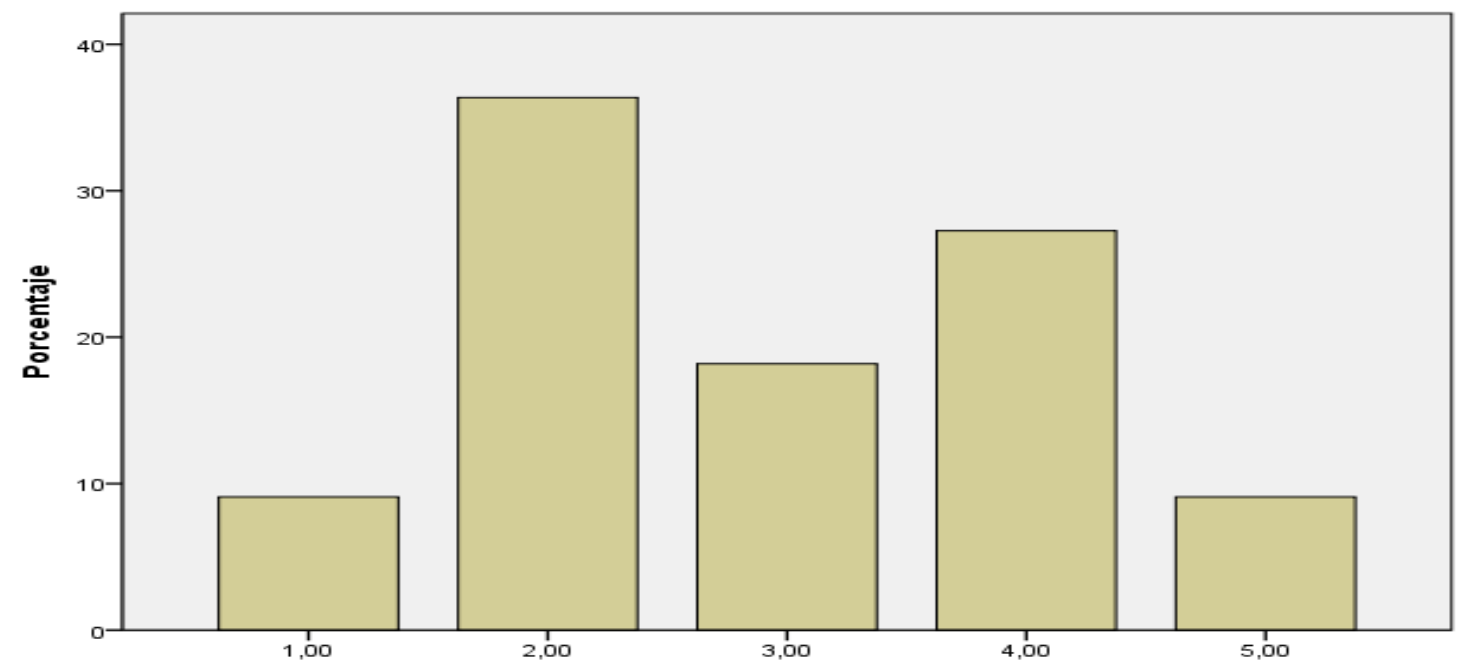

Gráfico 3. Dimensión Posinstruccionales.

En este renglón los valores que muestran la Tabla 7 y el Gráfico 3 deben llamar seriamente a la reflexión, puesto que se obtuvo sólo el $36,4 \%$ de respuestas consideradas como favorables, resultado muy inferior al $80 \%$ establecido como mínimo en el criterio de decisión para refrendar la existencia de una tendencia favorable en el uso de estrategias vinculadas con esta dimensión. Por tanto, los docentes encuestados muestran notables deficiencias en la utilización de estrategias didácticas referidas a la dimensión Postinstruccionales.
Estableciendo una diferencia con lo expuesto por Villarruel (2009) nos presenta esta estrategia para promover y potenciar enlaces adecuado entre los conocimientos previos y la información nueva asegurando un mayor significado en los aprendizajes. Lo anterior permite expresar que a las docentes les está haciendo falta este componente para evidenciar un verdadero aprendizaje significativo en el aula.

Lo que resulta que las docentes de preescolar de la institución objeto de 
estudio no consideran relevante que a los niños y niñas se les apliquen estrategias para finalizar su momento de clase de modo que logren un mayor significado en los aprendizajes lo que no les permita organizar correctamente la información nueva que se les ha presentado.

Al observar en detalle el comportamiento de las tres dimensiones, es importante resaltar un hallazgo en apariencia contradictorio: El uso de las estrategias didácticas preinstruccionales y las posinstruccionales (previas y posteriores a las sesiones de enseñanza y aprendizaje) por parte de los docentes sujetos de estudio presenta deficiencias significativas, mientras que en la aplicación de las estrategias didácticas coinstruccionales (que son aquellas que se usan de manera continua durante el desarrollo de una sesión o secuencia educativa) los docentes muestran un alto desempeño.

$\mathrm{Si}$ retomamos los preceptos de Ausubel (1983), respecto a las condiciones que se debe dar para que haya un aprendizaje significativo en lo referente al significado psicológico del material del contenido que se va a enseñar, que se refiere a la presentación organizada de dicho material y en estrecha relación con la experiencia cognoscitiva que tiene en particular cada estudiante se puede decir que sin la presencia o el uso de estrategias preinstrucionales y posinstrucionales los contenidos pierden su intencionalidad y potencial como material significativo para los niños y niñas, con esto entendemos que lo que se va a aprender solo mantiene un significado lógico. Lo anterior nos lleva a concluir que entre las docentes de la institución objeto de estudio no hay un amplio conocimiento sobre las estrategias, sus diferencias e intencionalidad de uso en cada uno de los momentos o etapas de sus clases e igualmente la importancia de utilizarlas desde el nivel preescolar, como base para un futuro aprendizaje $\mathrm{o}$ un buen desempeño de sus estudiantes cuando ingresen al nivel básico primario.

\section{Variable Estrategias para el aprendizaje significativo.}

Tabla 8. Variable Estrategias para el Aprendizaje Significativo

\begin{tabular}{cccc}
\hline Categoría de Respuesta & Frecuencia & Porcentaje & $\begin{array}{c}\text { Porcentaje } \\
\text { Acumulado }\end{array}$ \\
\hline 2.00 & 3 & 27.3 & 27.3 \\
3.00 & 2 & 18.2 & 45.5 \\
4.00 & 5 & 45.5 & 90.9 \\
5.00 & 1 & 9.1 & 100.0 \\
Total & 11 & 100.0 & \\
\hline
\end{tabular}

Fuente: SPSS aplicado a la base de datos (2017). 


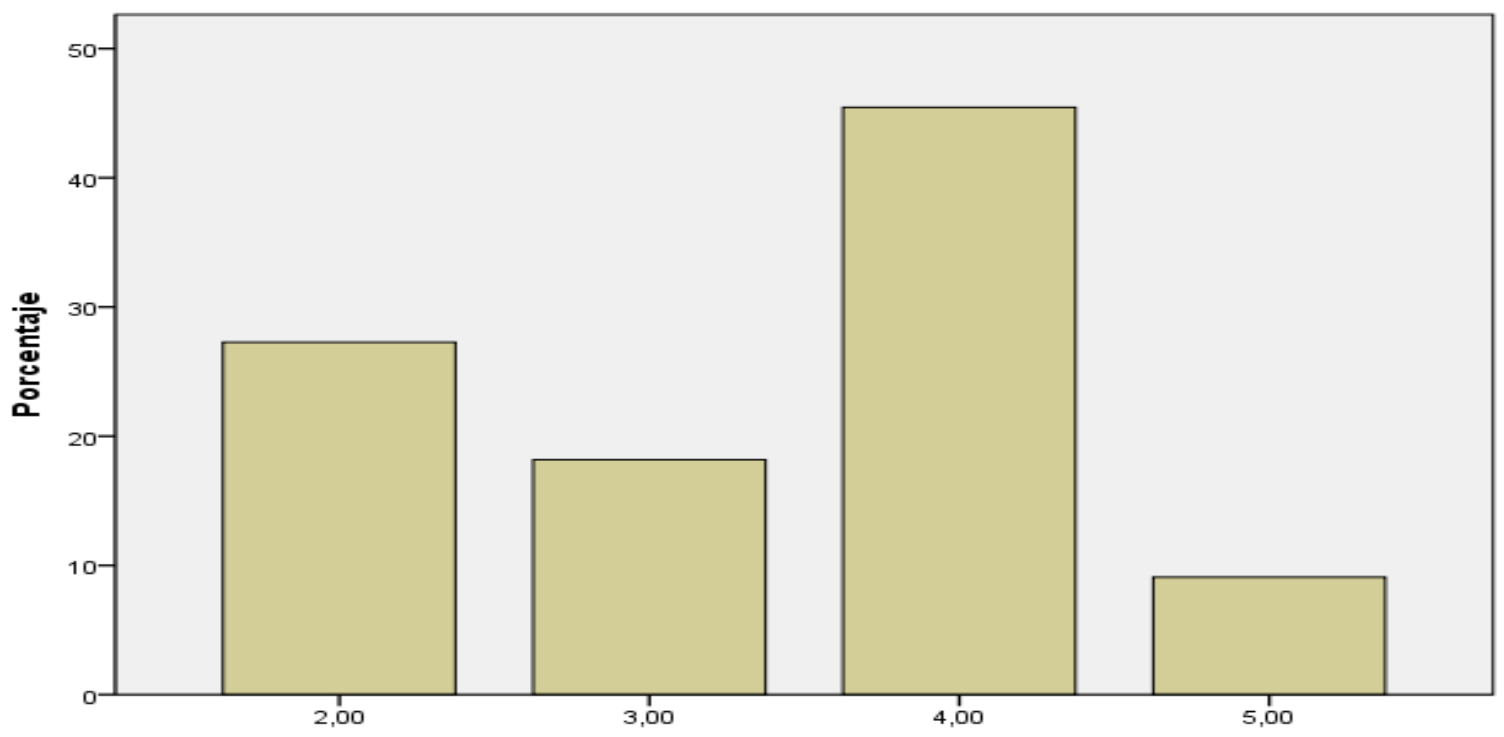

Gráfico 4. Variable estrategias para el aprendizaje significativo.

En cuanto al comportamiento general, para la variable objeto de estudio de esta investigación, los resultados de la Tabla 8 y el gráfico 4 exhiben un porcentaje de respuestas favorables del $54,5 \%$. En consecuencia, no se evidencia una tendencia favorable de desempeño por parte de los docentes del grupo de estudio referida al uso o implementación de estrategias para el aprendizaje significativo.

La implementación de estrategias para el aprendizaje significativo por parte de las docentes del nivel preescolar que fueron encuestadas, presenta deficiencia. Esto contrasta con lo expresado por Díaz Barriga y Hernández (2002) quienes manifiestan que el docente debe contar con un amplio conocimiento de estrategias, teniendo claro sus funciones y la manera apropiada de utilizarlas, con la intención de facilitar el aprendizaje significativo de sus estudiantes. Consideran también que deberán ser aplicadas de manera flexible y adaptable, teniendo en cuenta el dominio del conocimiento, contextos o secuencia de enseñanza de que se trate.

Esto lleva a reflexionar si realmente en las prácticas pedagógicas de dichas docentes se está potenciando un aprendizaje verdaderamente significativo pues al no utilizar estrategias preinstrucionales y posinstrucionales se puede concluir que lo aprendido en muchas ocasiones carece de significación para los niños y las niñas y que lo aprendido se está limitando a actividades motrices, memorísticas y repetitivas sin proyección al campo de la educación básica primaria. 


\section{CONCLUSIONES}

La presente investigación se realizó en la IED Rodrigo de Bastidas, una institución de carácter oficial que ofrece sus servicios educativos a estudiantes desde la edad preescolar hasta el grado 11 de Educación Media. El proceso central del mismo tomó como referente a las docentes del nivel preescolar, las cuales laboran con niños y niñas de cinco años de edad, provenientes de barrios aledaños de estratos socio económico bajo y quienes han recibido atención previa en hogares de bienestar familiar, que ofrecen atención básica a la primera infancia.

La problemática abordada en este trabajo se basó en el análisis del tipo de estrategias que utilizan las docentes del nivel preescolar, durante el desarrollo de sus clases a fin de lograr un aprendizaje significativo en sus estudiantes. Dicho análisis parte de la premisa en relación a los bajos resultados académicos que muestran los niños que posteriormente cursan el primer grado de educación básica en la misma institución.

Para continuar el proceso de investigación, se aplicó un instrumento de recolección de información, consistente en un cuestionario de 39 preguntas dirigidas a las docentes, el cual pretendía indagar sobre las estrategias que utilizaban las docentes antes, durante y después de abordar una temática determinada. Las preguntas aplicadas se sustentaron en un referente teórico relacionado con los tipos de estrategias: preinstruccionales, coinstruccionales y postinstruccionales desde el postulado de diferentes autores.

Con relación al resultado de la encuesta se puede decir que las Estrategias para el aprendizaje significativo utilizadas por las docentes de preescolar de la institución educativa objeto de estudio, en cuanto a la dimensión preinstruccional se evidenció que en la actualidad existe una marcada debilidad en cuanto a la indagación de los conocimientos previos que traen los niños y niñas sobre las temáticas a tratar, pues este tipo de estrategias son aplicadas con poca frecuencia, perdiendo así su intencionalidad pedagógica. Razón a que las docentes no están disponiendo de manera efectiva las estrategias preinstruccionales.

Con respecto a las estrategias coinstruccionales, los resultados muestran que estas se utilizan con mayor frecuencia, lo que indica que las docentes atribuyen mayor importancia a las mismas pues tal vez consideran que son las más importantes para generar conocimientos o son suficientes para lograr aprendizaje significativo en sus estudiantes.

En cuanto a las estrategias posinstruccionales las cuales permiten afianzar y evaluar los contenidos presentados por las docentes de una manera más clara, por su poco uso, no permiten aprovechar el potencial que 
puede ofrecer la participación de los estudiantes y sus aportes cognitivos en pos de su propio aprendizaje.

En general se evidenciaron una serie de limitantes que se presentan en el manejo de las estrategias, por ello las docentes se encuentran desprovistas de un verdadero apoyo integral para el logro de los objetivos planteados, ya que la deficiencia en el uso de las estrategias origina un manejo inadecuado de la información previa que lleva el estudiante al aula, para lograr que la misma se asuma como el punto de partida a los propósitos educativos que establecen las mismas docentes.

De acuerdo a lo anteriormente expuesto, esta investigación pudiese generar en las docentes y en la misma institución educativa un interés o la necesidad de profundizar en el análisis de las estructuras pedagógicas organizadas en los planes de estudios, especialmente del nivel preescolar, así mismo tengan la posibilidad de replantear la manera de planificar y realizar sus clases, incluyendo elementos más significantes para los estudiantes, las mismas docentes y que sean acordes con las exigencias de la sociedad actual.

\section{REFERENCIAS}

Alvarado, S., Suárez J., Martha C. (2011). Transacciones educativas en primera infancia, una mirada desde las políticas públicas en Colombia: avances y desafíos. Recuperado de http://portal.oas.org/LinkClick.aspx
?fileticket=aibSqqumQR8\%3D\&tabi $\mathrm{d}=1317$ \&language $=$ es

Ausubel, D., Novak, J., Helen, H. (1983). Psicología Educativa: Un punto de vista cognoscitivo. $2^{\circ} \mathrm{Ed}$. México: Trillas

Campos C, Y. (2000). Estrategias de enseñanza aprendizaje. Recuperado de http://asec.org.mx/curso/wpcontent/uploads/2011/10/Estrategia s_aprendizaje_blog.pdf

Díaz- Barriga, A., Frida y Hernández R., G. (1998). Estrategias docentes para un aprendizaje significativo, México: Mc Graw-Hill/Interamericana Editores S.A

Díaz- Barriga, A., Frida y Hernández R., G. (2002). Estrategias docentes para un aprendizaje significativo. México. Mc Graw-Hill/Interamericana Editores S.A

Hernández S., R., Fernández C, C., Batista L, P. (2010). Metodología de la investigación, 5ta Ed. México: Mc Graw-Hill/Interamericana Editores

León, A. (2010). Principales razones del fracaso escolar en primer grado en Costa Rica. Ponencia preparada para el Tercer Informe Estado de la Educación. San José, Programa Estado de la Nación. Consejo Nacional De Rectores, Costa Rica, pp. 15-16 Recuperado de http://estadonacion.or.cr/files/biblio teca_virtual/educacion/003/Leon_2 010_fracaso_ escolar_primer_grado.pdf Mieles B, M., Henríquez L, I., y Sánchez, C, L. (2009). Identidad personal y profesional de los docentes de preescolar en el distrito de Santa Marta Recuperado de http://www.scielo.org.co/scielo.php ?script=sci_arttext\&pid=S012312942009000100005. Fecha de recuperación 28 de febrero del 2015 
Villarruel, J. (2009), La práctica educativa del maestro mediador, Revista Iberoamericana de Educación $\mathrm{n}^{\mathrm{o}}$ 50, Organización de Estados Iberoamericanos para la Educación, la Ciencia y la Cultura (OEI). México, $\quad \mathrm{P}$ 7-9. http://www.rieoei.org/deloslectores /2957Fuentes.pdf Fecha de recuperación 4 de abril del 2015 